\title{
EXPRESSION OF CYTOTOXIC T-LYMPHOCYTE ANTIGEN 2 ALPHA IN MOUSE BRONCHIOLAR EPITHELIAL CELLS: ANATOMICAL AND FUNCTIONAL IMPLICATIONS
}

Claudius Luziga

Correspondence to Dr. Claudius Luziga Department of Veterinary Anatomy\& Pathology, Sokoine

University of Agriculture, P.O. Box 3016 Morogoro, Tanzania. E-mail address: luziga@sua.ac.tz. Tel. +255 765 774033;

\begin{abstract}
The lung is one of the major sites of cathepsins $(B, H$, and $L)$ expression where they are involved in several functions including degradation of extracellular matrix proteins, remodelling of the lung tissue and processing of the invariant chain of the MHC class II complex. Under pathological conditions, cathepsins have been observed in inflammation, tumour progression and metastasis. The enzymatic activity of cathepsins is regulated by changes in $\mathrm{pH}$ and their interaction with inhibitors. One of the potent and specific inhibitors of cathepsin $L$ is the Cytotoxic T-lymphocyte antigen-2a (CTLA-2a) which was originally expressed in mouse activated T-cells and mast cells. However, the cellular localization and distribution pattern of CTLA-2a in the lung is not known. In this study, CTLA-2a expression in the lung was evaluated by immunofluorescence. Strong immunoreactivity was specifically detected in the bronchiolar epithelial cells of primary, secondary and terminal segments; moderate in the lymphoid nodules and scanty in the lung parenchyma. The distribution pattern implicates an important role of this inhibitor protein in relation to matrix remodelling, immune response and their relationship to lung development and diseases.
\end{abstract}

Keywords: CTLA-2a, immunofluorescence, mouse, lung

\section{INTRODUCTION}

The respiratory system evolved for efficient gas exchange with branching structures of trachea, bronchi, bronchioles and alveolar ducts that end as alveoli (Benkingsopp, 1967; Bowden, 1983). As opposed to trachea and bronchi, the bronchioles lack hyaline cartilage and they are divided into primary, secondary and tertiary or terminal bronchioles, which subdivide into two or more respiratory bronchioles and serve as regions of transition between conducting and respiratory portions of the respiratory system.

The mucosa of the primary and secondary bronchioles is thrown into longitudinal folds that decrease in height towards the end of the secondary bronchioles. The epithelium is simple columnar but cuboidal in terminal and respiratory bronchioles. Openings of alveolar ducts occur in respiratory bronchioles and they end up into alveoli where gas exchange takes place (Becci, et al., 1978).

The main cell types composing the bronchioles are ciliated cells, few microvillar cells, club cells (previously known as Clara cells) and clusters of neuroendocrine cells (DiAugustine and Sonstegard, 1984; Perl et al., 2005). The club cells secrete proteins, which serve as defence barrier and show anti-inflammatory, immunemodulating and anti-tumorigenic roles in the lung (Yang, et al., 2004; Lee et al., 2006; Rawlins et al., 2009; Reynolds, et al., 2010). Following injury to mouse bronchioles, club cells act as progenitor cells for maintenance of the epithelium in the steady-state and regeneration of the injured bronchiolar 
epithelium by differentiating into new ciliated cells (Hong, et al., 2001). Club cells are also reported to secrete a number of products including a surfactant which reduce surface tension that help to prevent collapse of the bronchioles; a cytochrome P-450 enzyme (CYP4B1) from smooth endoplasmic reticulum that detoxifies potentially harmful compounds in air (Stanley et al., 1992; Boei, et al., 2017); enzymes that break down mucus locally; molecules that participate in the transfer of IgA into the bronchiolar lumen and cytokines that regulate local inflammatory responses (Arsalane, et al., 2000; Singh and Katyal, 2000). On the other hand, the neuroendocrine cells produce serotonin, calcitonin gene-related peptide and the mitogen bombesin. These bioactive substances have a role in the redistribution of pulmonary blood flow, regulation of bronchomotor tone, modulation of the immune response, stimulation of sensory nerve fibers and regulation of lung growth and development (Van Lommel, 2001).
In the lung, cathepsins $(B, H$, and $L)$ are also shown to participate in inflammatory responses, degradation of extracellular matrix proteins, remodeling and processing of secreted proteins and chain of the MHC class II complex (Bertini et al., 1981; Nakagawa et al., 1998; Shi et al., 1999; Bühling, et al., 2004; Conus and Simon, 2008). Cathepsin L differs from cathepsins B and $\mathrm{H}$ in its striking capacity to hydrolyze proteins (Katunuma and Kominami, 1983). Interestingly, the structure of CTLA-2a is homologous to the proregion of cathepsin $L$ and that CTLA-2a is a potent and selective inhibitor of cathepsin L (Kurata et al. 2003). However, the cellular localization and distribution pattern of CTLA-2a in the lung is not known. The purpose of this study was therefore to examine by immunofluorescence the cellular localization and distribution pattern of CTLA-2a in the respiratory system. Such information is thought to be pertinent to several aspects of lung growth and diseases.

\section{MATERIALS AND METHODS}

\section{Tissue preparation}

All experiments conformed to the guidelines for animal use for experimentation of Sokoine University of Agriculture. Ten adult mice (five males and five females) were used in this study. The mice were deeply anesthetized with sodium pentobarbital $(60 \mathrm{mg} / \mathrm{kg})$ by intraperitoneal injection. Transcardiac perfusion with $0.01 \mathrm{M}$ phosphate-buffered saline (PBS; $\mathrm{pH}$ 7.4) was performed followed by $4 \%$ paraformaldehyde (PFA; Sigma-Aldrich, St. Louis, MO) in 0.1 phosphate buffer (PB; $\mathrm{pH}$ 7.4). Lung tissues were dissected and postfixed in $4 \%$ PFA for 2 hours at $4^{\circ} \mathrm{C}$ followed by processing in ascending ethanol series to paraffin wax. Tissue blocks were cut at $4 \mu \mathrm{m}$ thick to produce serial tissue sections. Some sections were used for Hematoxyline and Eosin staining and others for immunofluorescence labelling.

\section{Routine Hematoxyline and Eosin staining}

Tissue sections were deparaffinized in xylene followed by rehydration through a descending ethanol series to phosphate-buffered saline (0.01M PBS-pH7.4). The sections were stained using Hematoxylin-Eosin (H\&E) in order to examine the normal structures of the lung.

\section{Antibody generation}

First, production of the recombinant protein of the CTLA-2a molecule with N-terminal polyhistidine tag was performed followed by immunization of rabbit to obtain antiserum. Immunization and preparation of antiserum was performed as previously described (Takahashi et al., 1993). The polyclonal CTLA-2a antibody against CTLA-2a protein was obtained through affinity chromatography column with recombinant CTLA-2a conjugated resin. The 
specificity of the purified antibody was characterized by Western blot as reported previously (Luziga et al., 2008)

\section{Immunofluorescence analysis}

Deparaffinazation of the tissues was performed as described in H\&E staining. Tissue sections were incubated for $30 \mathrm{~min}$ at room temperature (RT) in $0.3 \% \mathrm{v} / \mathrm{v}$ hydrogen peroxide diluted in distilled water to inhibit endogenous peroxidase activity followed by washing ( $3 \times 5 \mathrm{~min}$ ) in PBS. Sections were then incubated with $10 \%$ goat normal serum for $30 \mathrm{~min}$ at RT to block nonspecific binding, followed by incubation with the
CTLA-2a antibody diluted at 1:500 in PBS, for 24 hours in a dark, humid chamber at $4^{\circ} \mathrm{C}$. For negative control, PBS was applied in place of CTLA-2a antibody. Sections were then washed (3X5min) in PBS followed by incubation with Alexa Fluor $®$ 488-conjugated chicken antirabbit IgG (FITC) at a dilution of 1:100 (Molecular Probes) for 1hour at RT followed by washing the sections (3X5min) in PBS and mounting. Immunolabelling was analyzed using Olympus $\mathrm{BH}-2$ microscope fitted with Olympus camera.

\section{RESULTS}

\section{Routine histological examination findings}

The histological structure of the conducting airways and respiratory region were of normal morphological organization. Description is focused on bronchioles in which strong positive immunofluorescence labelling for CTLA-2a protein was observed. By $\mathrm{H} \& \mathrm{E}$ the bronchioles possessed primary, secondary and terminal or tertiary segments with mucosa being thrown into longitudinal folds that decreased in height towards the end of the secondary bronchioles. The epithelium was simple columnar in primary and secondary bronchioles but cuboidal in terminal and respiratory bronchioles. Goblet cells were few in all segments but hyaline cartilage was absent.
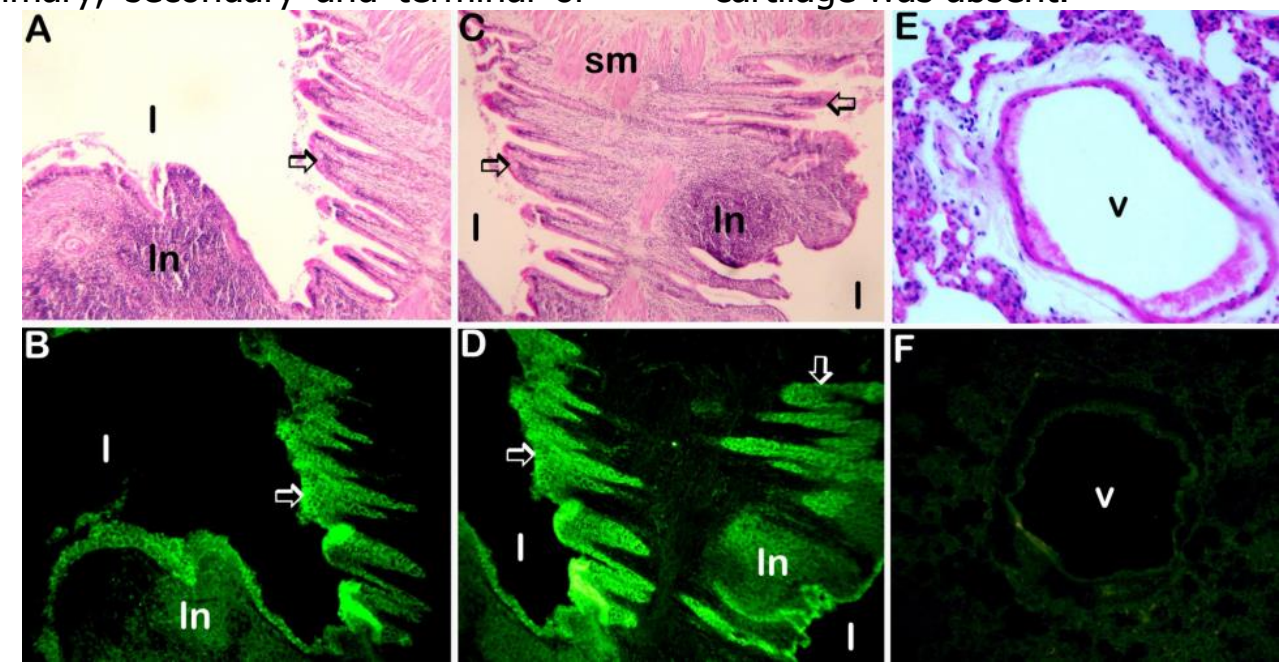

Fig. 1: Serial sections of primary bronchioles analysed by H\&E and Immunofluorescence for CTLA-2a protein. The first row shows normal histological organization of bronchioles (A\&C) and veins (E) stained with H\&E. Note the folded epithelium (arrows) due to contraction of smooth muscles (sm) and lack of hyaline cartilage in the bronchioles; I, indicate lumen of bronchioles and In, lymphatic nodules. The second row illustrates CTLA-2a expression in the primary bronchioles (B\&D) detected by immunofluorescence. Strong immunolabelling is seen in the epithelium of the primary bronchioles (arrows). CTLA-2a expression is absent in the lung parenchyma and wall of veins (F); I indicates lumen of bronchioles; In, lymphatic nodules and v, veins (Original magnification: X100). 
Few smooth muscle cells surrounding the bronchioles were also evident, which in turn were surrounded by loose connective tissue. Alveolar ducts, alveolar sacs that ended in alveoli were also in normal histological organization.

\section{Immunofluorescence analysis}

To investigate the cellular localization and distribution pattern of CTLA-2a protein, immunofluorescence analysis was performed on the lung tissue sections. Highest level of CTLA$2 a$ labeling was detected within bronchiolar epithelial cells in the primary, secondary, terminal and respiratory segments. Moderate immunoreactivity was seen in the lymphoid nodules that are composed of immune cells. The lung parenchyma, blood vessels including arteries and veins were virtually devoid of CTLA-2a protein.
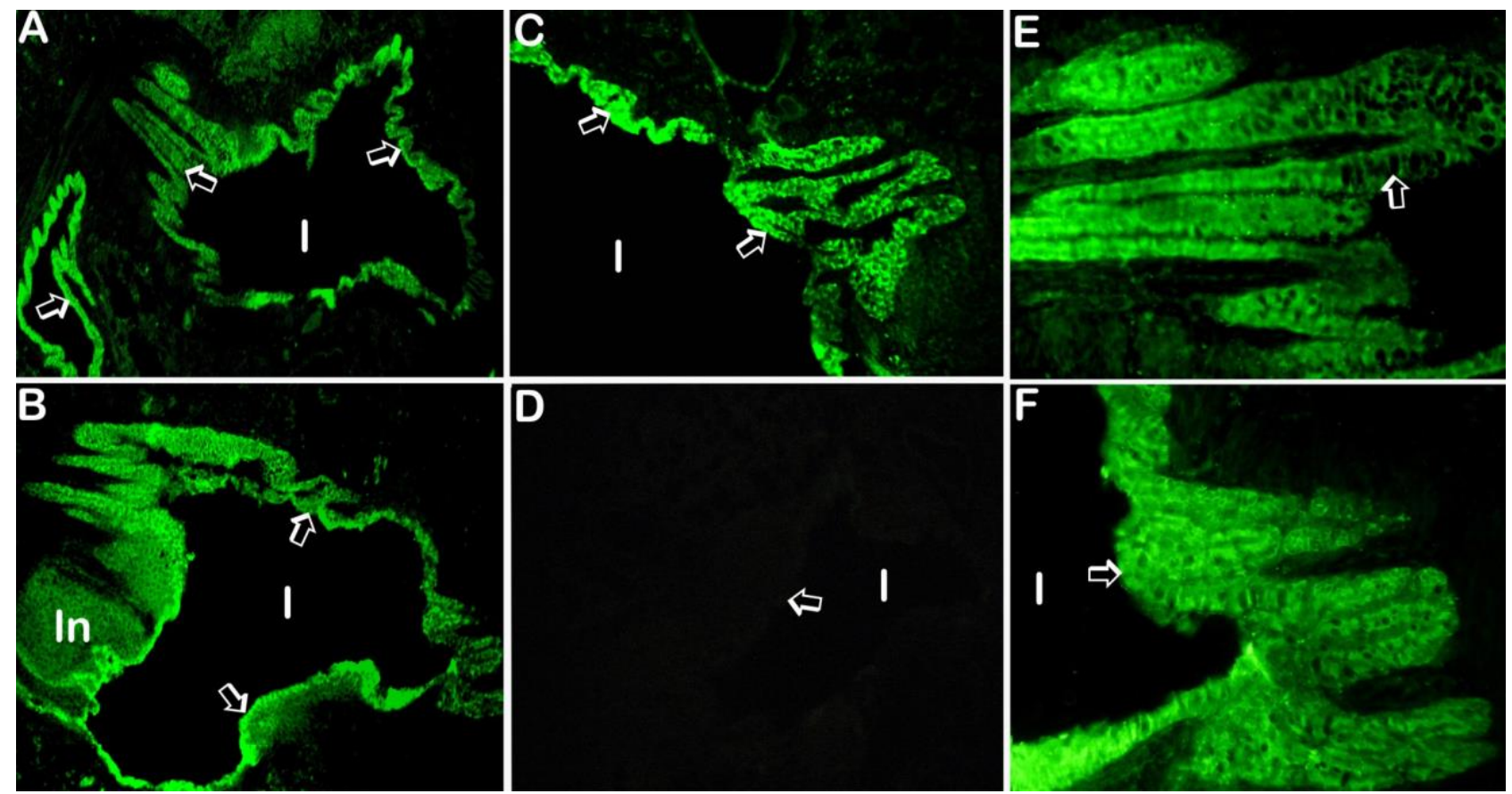

Fig. 2: Immunofluorescence labelling for CTLA-2a in the primary bronchioles. Plates A, B, C, E \& F show high level of CTLA-2a protein immunoreactivity in the epithelium of primary bronchioles (arrows). E \&F are higher magnification to show the localization of CTLA-2a protein in the bronchiolar epithelial cells. Labelling is virtually absent in the control sections (D) incubated with the 10\% PBS. I indicates lumen of the bronchioles and In, lymphatic nodules (Original magnification: $A, B, C \& D$ X100; E\&F X400). 

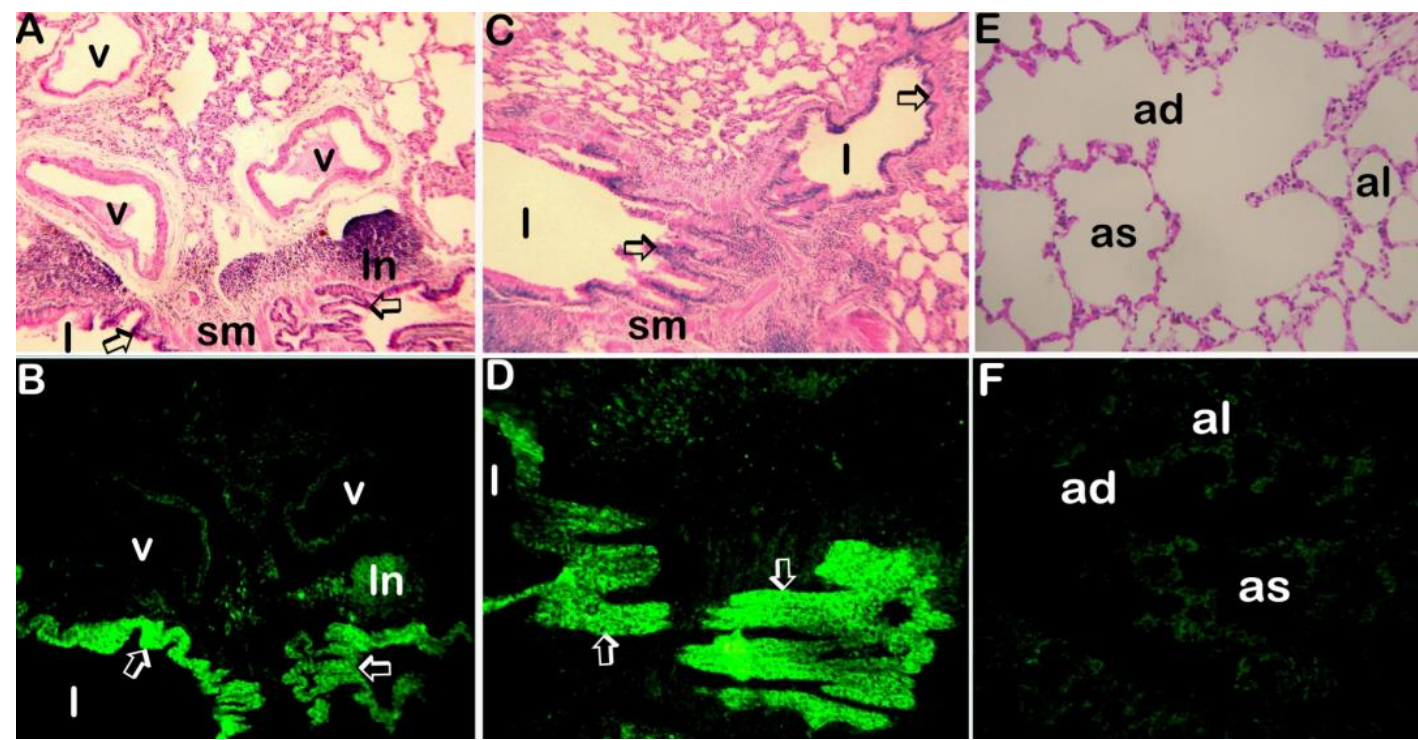

Fig. 3: Serial sections of secondary bronchioles and lung parenchyma analysed by H\&E and immunofluorescence for CTLA-2a protein. The first row (A\&C) demonstrates normal structures of secondary bronchioles with folded epithelium (arrows) and lack of hyaline cartilage and (E) normal lung parenchyma stained with H\&E. Note the normal histological organization (E) of alveolar duct (ad) with branching continuity to alveolar sac (as) and alveoli (al); I indicates lumen of bronchioles; In, lymphatic nodules and $\mathrm{V}$, veins. The second row (B\&D) shows secondary bronchioles and (F) lung parenchyma analyzed by immunofluorescence for CTLA-2a expression. Strong immunolabeling is seen in secondary bronchiolar epithelium (arrows), moderate in the lymphatic nodules (In) but not evident in the lung parenchyma ( $F$ ). (Original magnification: $A-F, X 100)$.
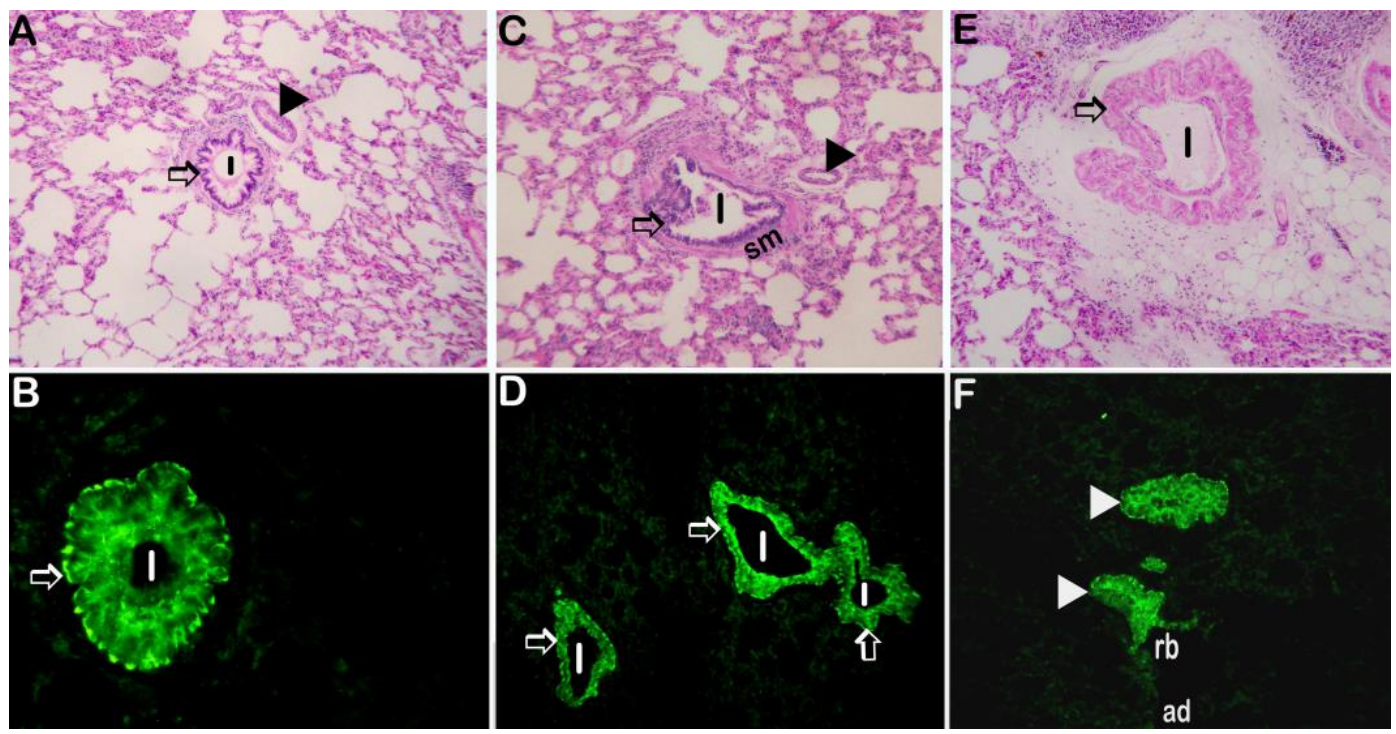

Fig. 4: Serial sections of terminal and respiratory bronchioles analysed by H\&E and immunofluorescence for CTLA2a protein. The first row (A,C and $\mathbf{E}$ ) shows normal structure of terminal or tertiary bronchiolar epithelium (arrows) stained with H\&E. Note the folded epithelium with reduced lumen (I) and the associated blood vessels (solid arrow heads), presence of smooth muscles and lack of hyaline cartilage. The second row (B\&D) shows immunofluorescence localization of CTLA-2a protein in the terminal bronchioles. High level of immunolabeling is seen in the epithelium (arrows) but not evident in the lung parenchyma. Note also positive immunoreactivity in the respiratory bronchioles (F) within the epithelial cells (solid arrow head). The respiratory bronchioles continue (rb) to alveolar duct (ad) which branch into alveolar sac (Original magnification: A-F, X100). 
Anatomy Journal of Africa. 2018. Vol 7 (2):1284 - 1291

\section{DISCUSSION}

The principal aims of this study were to investigate by immunofluorescence microscopy the cellular localization and distribution pattern of CTLA-2 $a$ in the adult mouse lung and deduce its functional implications in the respiratory system. Results obtained showed that CTLA-2a is highly expressed in the bronchiolar epithelium, moderate in the lymphatic nodules and virtually absent in the lung parenchyma. Whether the localization of CTLA-2a to bronchiolar epithelial cells is related to its role at least in part, to environmental components and its up-regulation relationship to contact of epithelial cells with the extracorporeal space or processing bronchiolar surfactant or other bioactive molecules or modulation of cathepsins degradation or to a novel function, is a question that remains to be clarified.

Studies show that the structure of CTLA-2a protein is highly homologous to the proregion of cathepsins. The propeptide of the proregion is part of the enzyme but CTLA-2a is a protein expressed independently and it is designated as a member of I29 protease inhibitor family (MEROPS peptidase database number I29.002 (http://merops.sanger.ac.uk/). CTLA-2a is also shown to exhibit potent and selective inhibition to mouse cathepsin L (Kurata et al. 2003). Although the functional implications underlying the localization of CTLA-2a protein in the bronchioles is unclear, in light of these, it is reasonable to conclude that through regulation of cathepsin activity, CTLA-2a in the lung participate in inflammatory responses, degradation of extracellular matrix proteins, remodelling and processing of secreted proteins. Indeed, simultaneous localization of cathepsin $L$ and CTLA-2a proteins in various regions of the mouse brain has been demonstrated by immunofluorescence analysis (Luziga, et al., 2016), suggesting that the turnover of CTLA-2a and cathepsin $L$ is regulated independently possibly through posttranslational regulation that allows immediate and selective changes in the protein levels in the bronchiolar epithelium.
One of the cell types composing the bronchiolar epithelium are neuroendocrine and club cells (DiAugustine and Sonstegard, 1984; Perl et al., 2005). Neuroendocrine cells produce serotonin, calcitonin gene-related peptide and the mitogen bombes that have a role in stimulation of sensory nerve fibers and regulation of lung growth and development (Van Lommel, 2001). Nerve fibers are also remarkable for their high concentration of CTLA-2a protein (Luziga, et al., 2008, 2016). On the other hand, club cells secrete proteins such as Scgb1a1/CCSP/CC10, which serve as a defence barrier and show antiinflammatory, immune-modulating and antitumorigenic roles in the lung (Yang, et al., 2004; Lee et al., 2006; Reynolds, et al., 2010). The cells also secrete other products such as surfactant which reduce surface tension in order to maintain bronchiolar patency and cytochrome P-450 enzyme (CYP4B1) that detoxifies potentially harmful compounds in air (Stanley, et al., 1992|, Boei, et al., 2017). Taking altogether, the localization of CTLA-2a at high levels in the bronchiolar epithelium is suggestive of its participation in the processing of bronchiolar surfactant and other enzymes or possibly involved in some other novel biological functions that are yet to be identified.

In conclusion, this study demonstrates that the expression of CTLA-2a protein in the adult mouse lung is mainly localized in bronchiolar epithelial cells. This finding is suggestive of an important function of CTLA-2a in relation to matrix remodeling, immune response and their relationship to lung diseases. This report is thought to be a helpful tool for understanding CTLA-2a and opens the way to new studies on the function of CTLA-2a in the respiratory system.

Acknowledgements:

Grateful acknowledgement goes to the Japanese Ministry of Education, Culture, Sports, Science and Technology for financial support. 


\section{REFERENCES}

1. Arsalane K, Broeckaert F, Knoops B, Wiedig M, Toubeau G, Bernard A. 2000. Clara cell specific protein (CC16) expression after acute lung inflammation induced by intratracheal lipopolysaccharide administration. Am J Respir Crit Care Med. 161:1624-1630.

2. Becci PJ, McDowell EM, Trump BF. 1978. The respiratory epithelium. II. Hamster trachea, bronchus, and bronchioles. J Natl Cancer Inst. 61:551-61.

3. Bertini F, Mayorga L, Gonzalez M. 1981. The development of lysosomal apparatus. II. Incorporation, subcellular distribution, and intraparticulate hydrolysis of $131 \mathrm{I}$-albumin by liver of mice at perinatal stages. J Cell Physiol. 109:281-287.

4. Blenkinsopp, W. K.1967. Proliferation of respiratory tract epithelium in the rat. Exp Cell Res 46:144-154.

5. Boei JJWA, Vermeulen S, Klein B. et al. 2017. Xenobiotic metabolism in differentiated human bronchial epithelial cells. Arch Toxicol. 91:2093-2105.

6. Bowden DH. 1983. Cell turnover in the lung. Am Rev Respir Dis. 128:46-48.

7. Bühling F, Waldburg N, Reisenauer A, Heimburg A, Golpon H, Welte T. 2004. Lysosomal cysteine proteases in the lung: role in protein processing and immunoregulation. Eur Respir J. 23:620-628.

8. Conus S, Simon HU. 2008 Cathepsins: key modulators of cell death and inflammatory responses. Biochem Pharmacol. 76: 1374-1382.

9. DiAugustine RP, Sonstegard KS.1984. Neuroendocrinelike (small granule) epithelial cells of the lung. Environ Health Perspect. 55:271-295.

10. Hong KU, Reynolds SD, Giangreco A, Hurley CM, Stripp BR. 2001. Clara cell secretory proteinexpressing cells of the airway neuroepithelial body microenvironment include a label-retaining subset and are critical for epithelial renewal after progenitor cell depletion. Am J Respir Cell Mol Biol. 24:671-681.

11. Katunuma N, Kominami E. 1983. Structures and Functions of Lysosomal Thiol Proteinases and Their Endogenous Inhibitor. Current Topics in Cellular Regulation. 22:71-101.

12. Kurata M, Hirata M, Watabe S, Miyake M, Takahashi SY, Yamamoto Y. 2003. Expression, purification, and inhibitory activities of mouse cytotoxic T-lymphocyte antigen-2. Protein Expression and Purification. 32:119-125.

13. Lee YC, Zhang Z, Mukherjee AB. 2006. Mice lacking uteroglobin are highly susceptible to developing pulmonary fibrosis. FEBS Letters. 580:4515-4520.

14. Luziga C, Nakamura O, Deshapriya RMC, Usui M, Miyaji M, Wakimoto M, Wada N, Mbassa G, Yamamoto Y. 2008. Dendritic andaxonal localization of cytotoxic T-lymphocyte antigen-2 alpha protein in mouse brain. Brain Res. 1204:40-52.

15. Luziga C, Nga BT, Mbassa G, Yamamoto Y. 2016. Cathepsin L coexists with Cytotoxic Tlymphocyte Antigen-2 alpha in distinct regions of the mouse brain. Acta Histochem. 118:704-710.

16. Nakagawa T, Roth W, Wong P, Nelson A, Farr A, Deussing J, Villadangos JA, Ploegh H, Peters C, Rudensky AY. 1998. Cathepsin L: critical role in Ii degradation and CD4 T cell selection in the thymus. Science.280:450-453.

17. Perl AK, Wert SE, Loudy DE, Shan Z, Blair PA, et al. 2005. Conditional recombination reveals distinct subsets of epithelial cells in trachea, bronchi, and alveoli. Am J Respir Cell Mol Biol. 33:455-462.

18. Rawlins EL, Okubo T, Xue Y, Brass DM, Auten RL, Hasegawa H, et al. 2009. The role of Scgb1a1+ Clara cells in the long-term maintenance and repair of lung airway, but not alveolar, epithelium. Cell Stem Cell. 4:525-534. 
19. Rawlins EL, Okubo T, Xue Y, Brass DM, Auten RL, Hasegawa H, et al. The role of Scgb1a1+ Clara cells in the long-term maintenance and repair of lung airway, but not alveolar, epithelium. Cell Stem Cell. 2009;4(6):525-534. doi: 10.1016/j.stem.2009.04.002 [PMC free article] [PubMed]

20. Reynolds SD, Malkinson AM. 2010. Clara cell: progenitor for the bronchiolar epithelium. Int J Biochem Cell Biol. 42:1-4.

21. Shi GP, Villadangos JA, Dranoff G, Small C, Gu LJ, Haley KJ, Riese R, Ploegh HL, Chapman HA. 1999. Cathepsin S required for normal MHC class II peptide loading and germinal center development. Immunity 10: 197-206.

22. Singh G, Katyal SL. 2000. Clara cell proteins. Ann N Y Acad Sci. 923:43-58.

23. Stanley LA, Carmichael J, Wolf CR. 1992. Cytochrome P-450 induction in human lung tumourderived cell lines. Characterisation and effects of inflammatory mediators. Eur $J$ Biochem. 208:521-529.

24. Takahashi SY, Yamamoto Y, Shionoya Y, Kageyama T. 1993. Cysteine proteinase from the eggs of the silkmoth, Bombyx mori: identification of a latent enzyme and characterization of activation and proteolytic processing in vivo and in vitro. J. Biochem. 144: 267-272.

25. Van Lommel A. 2001. Pulmonary neuroendocrine cells (PNEC) and neuroepithelial bodies (NEB): chemoreceptors and regulators of lung development. Paediatr Respir Rev. 2:171-176.

26. Yang Y, Zhang Z, Mukherjee AB, Linnoila RI.2004. Increased susceptibility of mice lacking Clara cell $10-\mathrm{kDa}$ protein to lung tumorigenesis by 4-(methylnitrosamino)-1-(3-pyridyl)-1-butanone, a potent carcinogen in cigarette smoke. J Biol Chem. 279:29336-29340. 